\title{
Computation of initial conditions and inputs for given outputs of fractional and positive discrete-time linear systems
}

\author{
TADEUSZ KACZOREK
}

\begin{abstract}
The problem of computation of initial conditions and inputs for given outputs of fractional standard and positive discrete-time linear systems is formulated and solved. Necessary and sufficient conditions for existence of solution to the problem are established. It is shown that there exist the unique solutions to the problem only if the pair $(A, C)$ of the system is observable.
\end{abstract}

Key words: computation, initial condition, observability, positive, discrete-time, linear, system

\section{Introduction}

In positive systems inputs, state variables and outputs take only non-negative values. Examples of positive systems are industrial processes involving chemical reactors, heat exchangers and distillation columns, storage systems, compartmental systems, water and atmospheric pollution models. A variety of models having positive linear behavior can be found in engineering, management science, economics, social sciences, biology and medicine, etc. An overview of state of the art in positive linear theory is given in the monographs $[2,3]$. The notions of controllability and observability and the decomposition of linear systems have been introduced by Kalman $[10,11]$. Those notions are the basic concepts of the modern control theory $[1,9,12,13,6]$. They have been also extended to positive linear systems $[2,3]$. The decomposition of the pair $(A, C)$ and $(A, B)$ of the positive discrete-time linear systems has been addressed in [4] and the problem of computation of initial conditions and inputs for given outputs of standard and positive discrete-time linear system in [7].

In this paper the problem of computation of initial conditions and inputs for given outputs of fractional standard and positive discrete-time linear systems will be formu-

The Author is with Bialystok University of Technology, Faculty of Electrical Engineering, Wiejska 45D, 15-351 Bialystok, Poland. E-mail: kaczorek@isep.pw.edu.pl

This work was supported by Ministry of Science and Higher Education in Poland under work S/WE/1/11.

Received 03.01.2012. 
lated and solved. Necessary and sufficient conditions for existence of solutions to the problem will be established.

The paper is organized as follows. In section 2 the problem is formulated. The main results of the paper are given in sections 3 and 4 . The necessary and sufficient conditions for existence of solutions to the problem for fractional standard systems are established in section 3 and for fractional positive system in section 4. Concluding remarks are given in section 4 .

The following notation will be used: $\mathfrak{R}$ - the set of real numbers, $\Re^{n \times m}$ - the set of $n \times m$ real matrices, $\Re_{+}^{n \times m}$ - the set of $n \times m$ matrices with nonnegative entries and $\Re_{+}^{n}=\Re_{+}^{n \times 1}, I_{n}-$ the $n \times n$ identity matrix.

\section{Preliminaries and problem formulation}

Consider the fractional discrete-time linear system [8]

$$
\begin{aligned}
& \Delta^{\alpha} x_{i+1}=A x_{i}+B u_{i} \\
& y_{i}=C x_{i}+D u_{i}
\end{aligned}, \quad i \in Z_{+}, \quad 0<\alpha<1
$$

where $\alpha$ is fractional order, $x_{i} \in \mathfrak{R}^{n}$ is the state vector, $u_{i} \in \mathfrak{R}^{m}$ is the input vector, $y_{i} \in \mathfrak{R}^{p}$ is the output vector and $A \in \mathfrak{R}^{n \times n}, B \in \mathfrak{R}^{n \times m}, C \in \mathfrak{R}^{p \times n}, D \in \mathfrak{R}^{p \times m}$. The fractional difference of the order $\alpha \in(0,1)$ is defined by

$$
\Delta^{\alpha} x_{i}=x_{i}+\sum_{j=1}^{i}(-1)^{j}\left(\begin{array}{c}
\alpha \\
j
\end{array}\right) x_{i-j}, \quad i \in Z_{+}
$$

where

$$
\left(\begin{array}{c}
\alpha \\
j
\end{array}\right)=\frac{\alpha(\alpha-1) \ldots(\alpha-j+1)}{j !}, j=1,2, \ldots
$$

Substitution of (2) into (1) yields

$$
x_{i+1}=A_{\alpha} x_{i}+\sum_{j=1}^{i} c_{j}(\alpha) x_{i-j}+B u_{i}, \quad i \in Z_{+}
$$

where

$$
A_{\alpha}=A+I_{n} \alpha, \quad, c_{j}(\alpha)=(-1)^{j}\left(\begin{array}{c}
\alpha \\
j+1
\end{array}\right)>0, \quad j=1,2, \ldots
$$

The solution of (3a) has the form [8]

$$
x_{i}=\Phi_{i} x_{0}+\sum_{k=0}^{i-1} \Phi_{i-k-1} B u_{i}
$$


and the matrix $\Phi_{i}$ can be computed from the formula

$$
\Phi_{i+1}=A_{\alpha} \Phi_{i}+\sum_{j=1}^{i} c_{j}(\alpha) \Phi_{i-j}, \quad \Phi_{0}=I_{n} .
$$

Definition 1 [8] The system (1) is called the (internally) positive fractional system if and only if $x_{k} \in \mathfrak{R}_{+}^{n}, y_{k} \in \mathfrak{R}_{+}^{p}, k \in Z_{+}$for any initial conditions $x_{0} \in \mathfrak{R}_{+}^{n}$ and all input sequences $u_{k} \in \Re_{+}^{m}, k \in Z_{+}$.

Theorem 1 [8] The fractional system (1) is positive for $0<\alpha<1$ if and only if

$$
A_{\alpha} \in \mathfrak{R}_{+}^{n \times n}, \quad B \in \mathfrak{R}_{+}^{n \times m}, \quad C \in \mathfrak{R}_{+}^{p \times n}, \quad D \in \mathfrak{R}_{+}^{p \times m} .
$$

Definition 2 [8] The positive fractional system (1) is called observable in $q(q \geqslant p n)$ steps if on the bases of knowledge of the input sequence $u_{k} \in \mathfrak{R}_{+}^{m}$ and the output sequence $y_{k} \in \mathfrak{R}_{+}^{p}$ for $k=0,1, \ldots, q-1$ it is possible to determine uniquely the initial conditions $x_{0} \in \mathfrak{R}_{+}^{n}$.

A column (row) of the form $a e_{i}\left(a e_{i}, T\right.$-denotes the transpose), $i=1, \ldots, n ; a>0$ is called monomial. A square matrix $A$ is called monomial if in each row and in each column only one entry is positive and the remaining entries are zero.

Theorem 2 [8] The positive fractional system (1) is observable in q steps if and only if the matrix

$$
O_{q}=\left[\begin{array}{c}
C \\
C \Phi_{1} \\
\vdots \\
C \Phi_{q-1}
\end{array}\right]
$$

contains $n$ linearly independent monomial rows.

The problem under considerations can be stated as follows.

Given the sequence of outputs $y_{0}, y_{1}, \ldots, y_{n}$ compute the initial condition $x_{0}$ and input sequence $u_{0}, u_{1}, \ldots, u_{n}$ for the fractional standard and positive system (1). The problem can be considered as a generalization of the observability problem of fractional standard and positive discrete-time linear systems.

The following two cases will be considered separately for fractional standard and positive systems:

Case 1. The matrix $D=0$.

Case 2. The matrix $D \neq 0$. 


\section{Problem solution for standard systems}

Substituting the solution (4) into (1) we obtain

$$
y_{i}=C \Phi_{i} x_{0}+\sum_{k=0}^{i-1} C \Phi_{i-k-1} B u_{k}+D u_{i}, \quad i \in Z_{+}
$$

If $D=0$ then using (8) for $i=1,2, \ldots, n-1$ we obtain

$$
H z=y
$$

where

$$
\begin{gathered}
H=\left[\begin{array}{ccccc}
C & 0 & 0 & \ldots & 0 \\
C \Phi_{1} & C B & 0 & \ldots & 0 \\
C \Phi_{2} & C \Phi_{1} B & C B & \ldots & 0 \\
\vdots & \vdots & \vdots & \ddots & \vdots \\
C \Phi_{n-1} & C \Phi_{n-2} B & C \Phi_{n-3} B & \ldots & C B
\end{array}\right] \in \mathfrak{R}^{p n \times(n+(n-1) m)}, \\
z=\left[\begin{array}{c}
x_{0} \\
u_{0} \\
u_{1} \\
\vdots \\
u_{n-2}
\end{array}\right] \in \mathfrak{R}^{n+(n-1) m}, \quad y=\left[\begin{array}{c}
y_{0} \\
y_{1} \\
y_{2} \\
\vdots \\
y_{n-1}
\end{array}\right] \in \mathfrak{R}^{p n} .
\end{gathered}
$$

Similarly if $D \neq 0$ then we obtain

$$
\bar{H} \bar{z}=y
$$

where

$$
\begin{gathered}
\bar{H}=\left[\begin{array}{cccccc}
C & D & 0 & \ldots & 0 & 0 \\
C \Phi & C B & D & \ldots & 0 & 0 \\
C \Phi_{2} & C \Phi_{1} B & C B & \ldots & 0 & 0 \\
\vdots & \vdots & \vdots & \ddots & \vdots & \vdots \\
C \Phi_{n-1} & C \Phi_{n-2} B & C \Phi_{n-3} B & \ldots & C B & D
\end{array}\right] \in \mathfrak{R}^{p n \times(m+1) n} \\
\bar{z}=\left[\begin{array}{c}
x_{0} \\
u_{0} \\
u_{1} \\
\vdots \\
u_{n-1}
\end{array}\right] \in \mathfrak{R}^{(m+1) n} .
\end{gathered}
$$


In the proofs we will use the following well-known Kronecker-Cappely Theorem [5].

Theorem 3 The equation (9a) has a solution $z$ for given $H$ and $y$ if and only if

$$
\operatorname{rank}\left[\begin{array}{cc}
H & y
\end{array}\right]=\operatorname{rank} H
$$

Case 1. $D=0$.

Theorem 4 Let $D=0$ and $n+(n-1) m \geqslant n p$. Then the equation (9a) has a solution $x_{0}, u_{0}, u_{1}, \ldots, u_{n-2}$ for any given sequence $y_{0}, y_{1}, \ldots, y_{n-1}$ if and only if

$$
\operatorname{rank} H^{\prime}=p n
$$

where

$$
H^{\prime}=\left[\begin{array}{ccccc}
C & 0 & 0 & \ldots & 0 \\
C A_{\alpha} & C B & 0 & \ldots & 0 \\
C A_{\alpha}^{2} & C A_{\alpha} B & C B & \ldots & 0 \\
\vdots & \vdots & \vdots & \ldots & \vdots \\
C A_{\alpha}^{n-1} & C A_{\alpha}^{n-2} B & C A_{\alpha}^{n-3} B & \ldots & C B
\end{array}\right]
$$

Moreover, the equation has the unique solution

$$
z=H^{-1} y
$$

if $n+(n-1) m=n p$ and many solutions if $n+(n-1) m>n p$.

Proof. From (5) we have

$$
\Phi_{1}=A_{\alpha}, \quad \Phi_{2}=A_{\alpha}^{2}+c_{1} I_{n}, \quad \Phi_{3}=A_{\alpha}^{3}+c_{1} A_{\alpha}+c_{2} I_{n}, \ldots
$$

where $c_{j}=c_{j}(\alpha)=(-1)^{j}\left(\begin{array}{c}\alpha \\ j+1\end{array}\right), j=1,2, \ldots$. Using (14) we can write the matrix $H$ as

$$
H=P H^{\prime}
$$

where

$$
P=\left[\begin{array}{cccccc}
I_{p} & 0 & 0 & \ldots & 0 & 0 \\
0 & I_{p} & 0 & \ldots & 0 & 0 \\
c_{1} I_{p} & 0 & I_{p} & \ldots & 0 & 0 \\
c_{2} I_{p} & c_{1} I_{p} & 0 & \ldots & 0 & 0 \\
\vdots & \vdots & \vdots & \ldots & \vdots & \vdots \\
c_{n-2} I_{p} & c_{n-3} I_{p} & c_{n-4} I_{p} & \ldots & 0 & I_{p}
\end{array}\right]
$$


From (15) it follows that

$$
\operatorname{rank} H=p n
$$

if and only if (12) holds. If the condition (12) is met then by Theorem 3 the equation (9a) has the solution $\mathrm{z}$ for any sequence $y_{0}, y_{1}, \ldots, y_{n-1}$. If additionally $(m+1) n-m<n p$ the matrix $H$ is square and the solution is unique. If $(m+1) n-m>n p$ the equation (9a) has many solutions.

Theorem 5 Let $D=0$ and $n p>(m+1) n-m$. Then the equation (9a) has a solution $x_{0}, u_{0}, u_{1}, \ldots, u_{n-2}$ for a given sequence $y_{0}, y_{1}, \ldots, y_{n-1}$ if and only if the condition

$$
\operatorname{rank}\left[\begin{array}{cc}
H^{\prime} & y
\end{array}\right]=\operatorname{rank} H^{\prime}
$$

is satisfied. Moreover, the equation has the unique solution if

$$
\operatorname{rank} H^{\prime}=(m+1) n-m
$$

and it has many solutions if

$$
\operatorname{rank} H^{\prime}<(m+1) n-m
$$

Proof By Theorem 3 the equation (9a) has a solution $z$ for any $y$ if and only if the condition (11) is satisfied. From (15) it follows that the condition (17) is equivalent to the condition (11). The solution is unique if (18) holds since the matrix $H^{\prime}$ has full column rank. If (17) and (19) hold then the equation (9a) has many solutions.

Theorem 6 The equation (9a) has the unique solution $z$ only if the pair $(A, C)$ is observable.

Proof The equation (9a) has the unique solution only if the matrix $H^{\prime}$ has full column rank and this implies the full column rank of the observability matrix of the pair $\left(A_{\alpha}, C\right)$, i.e.

$$
\operatorname{rank}\left[\begin{array}{c}
C \\
C A_{\alpha} \\
\vdots \\
C A_{\alpha}^{n-1}
\end{array}\right]=n .
$$

Example 1 Consider the fractional discrete-time system (1) for $\alpha=0.5$ and with the matrices

$$
A=\left[\begin{array}{ll}
1 & 2 \\
1 & 0
\end{array}\right], \quad B=\left[\begin{array}{l}
1 \\
0
\end{array}\right], \quad C=\left[\begin{array}{ll}
1 & 0
\end{array}\right], \quad D=[0] .
$$


Compute the initial condition $x_{0}=\left[\begin{array}{l}x_{01} \\ x_{02}\end{array}\right]$ and the input $u_{0}$ of the system for the given output sequence $y_{0}, y_{1}$. In this case we have $n=2, m=p=1,(m+1) n-m=3>n p=2$,

$$
A_{\alpha}=A+I_{n} \alpha=\left[\begin{array}{cc}
1.5 & 2 \\
1 & 0.5
\end{array}\right]
$$

and the matrix

$$
H=\left[\begin{array}{cc}
C & 0 \\
C A & C B
\end{array}\right]=\left[\begin{array}{lll}
1 & 0 & 0 \\
0 & 1 & 1
\end{array}\right]
$$

has the full row rank. The equation (9a) has the form

$$
\left[\begin{array}{ccc}
1 & 0 & 0 \\
1.5 & 2 & 1
\end{array}\right]\left[\begin{array}{l}
x_{01} \\
x_{02} \\
u_{0}
\end{array}\right]=\left[\begin{array}{l}
y_{0} \\
y_{1}
\end{array}\right]
$$

and it has many solutions for any sequence $y_{0}, y_{1}$. From (23) we have

$$
\left[\begin{array}{l}
x_{01} \\
x_{02}
\end{array}\right]=\left[\begin{array}{cc}
1 & 0 \\
1.5 & 2
\end{array}\right]^{-1}\left[\begin{array}{c}
y_{0} \\
y_{1}-u_{0}
\end{array}\right]=\left[\begin{array}{c}
2 y_{0} \\
y_{1}-1.5 y_{0}-u_{0}
\end{array}\right] \text { for arbitrary } u_{0}
$$

or

$$
\left[\begin{array}{c}
x_{01} \\
u_{0}
\end{array}\right]=\left[\begin{array}{cc}
1 & 0 \\
1.5 & 1
\end{array}\right]^{-1}\left[\begin{array}{c}
y_{0} \\
y_{1}-2 x_{02}
\end{array}\right]=\left[\begin{array}{c}
y_{0} \\
y_{1}-1.5 y_{0}-2 x_{02}
\end{array}\right] \text { for arbitrary } x_{02} .
$$

Example 2 Consider the fractional system (1) for $\alpha=0.6$ with the matrices

$$
A=\left[\begin{array}{ccc}
0.4 & 1 & 0 \\
0 & 0.2 & 1 \\
0 & 1 & 0.4
\end{array}\right], \quad B=\left[\begin{array}{l}
0 \\
0 \\
1
\end{array}\right], \quad C=\left[\begin{array}{lll}
1 & 0 & 0 \\
0 & 0 & 1
\end{array}\right], \quad D=\left[\begin{array}{l}
0 \\
0
\end{array}\right] .
$$

Using (14) and (24) we obtain

$$
\Phi_{1}=A_{\alpha}=A+I_{n} \alpha=\left[\begin{array}{ccc}
1 & 1 & 0 \\
0 & 0.8 & 1 \\
0 & 1 & 1
\end{array}\right], \quad \Phi_{2}=A_{\alpha}^{2}+c_{1} I_{n}=\left[\begin{array}{ccc}
1.12 & 1.8 & 1 \\
0 & 1.76 & 1.8 \\
0 & 1.8 & 2.12
\end{array}\right]
$$


and the matrix

$$
H=\left[\begin{array}{ccc}
C & 0 & 0 \\
C \Phi_{1} & C B & 0 \\
C \Phi_{2} & C \Phi_{1} B & C B
\end{array}\right]=\left[\begin{array}{ccccc}
1 & 0 & 0 & 0 & 0 \\
0 & 0 & 1 & 1 & 0 \\
1 & 1 & 0 & 0 & 0 \\
0 & 1 & 1 & 1 & 0 \\
1.12 & 1.8 & 1 & 0 & 0 \\
0 & 1.8 & 2.12 & 1 & 1
\end{array}\right]
$$

The matrix (25) has full column rank. Using elementary row operations [5] we can reduce the equation

$$
\left[\begin{array}{ccccc}
1 & 0 & 0 & 0 & 0 \\
0 & 0 & 1 & 1 & 0 \\
1 & 1 & 0 & 0 & 0 \\
0 & 1 & 1 & 1 & 0 \\
1.12 & 1.8 & 1 & 0 & 0 \\
0 & 1.8 & 2.12 & 1 & 1
\end{array}\right]\left[\begin{array}{l}
x_{01} \\
x_{02} \\
x_{03} \\
u_{0} \\
u_{1}
\end{array}\right]=\left[\begin{array}{l}
y_{01} \\
y_{02} \\
y_{11} \\
y_{12} \\
y_{21} \\
y_{22}
\end{array}\right]
$$

to the form

$$
\left[\begin{array}{ccccc}
1 & 0 & 0 & 0 & 0 \\
0 & 0 & 1 & 1 & 0 \\
0 & 0 & 0 & 0 & 0 \\
0 & 1 & 0 & 0 & 0 \\
1.12 & 1.8 & 1 & 0 & 0 \\
0 & 1.8 & 2.12 & 1 & 1
\end{array}\right]\left[\begin{array}{c}
x_{01} \\
x_{02} \\
x_{03} \\
u_{0} \\
u_{1}
\end{array}\right]=\left[\begin{array}{c}
y_{01} \\
y_{02} \\
y_{11}-y_{01}+y_{02}-y_{12} \\
y_{12}-y_{02} \\
y_{21} \\
y_{22}
\end{array}\right] .
$$

From (27) it follows that the condition (11) is met if and only if

$$
y_{11}-y_{01}+y_{02}-y_{12}=0 .
$$

Omitting the third equation in (27) we obtain

$$
\left[\begin{array}{ccccc}
1 & 0 & 0 & 0 & 0 \\
0 & 0 & 1 & 1 & 0 \\
0 & 1 & 0 & 0 & 0 \\
1.12 & 1.8 & 1 & 0 & 0 \\
0 & 1.8 & 2.12 & 1 & 1
\end{array}\right]\left[\begin{array}{c}
x_{01} \\
x_{02} \\
x_{03} \\
u_{0} \\
u_{1}
\end{array}\right]=\left[\begin{array}{c}
y_{01} \\
y_{02} \\
y_{12}-y_{02} \\
y_{21} \\
y_{22}
\end{array}\right]
$$


and

$$
\begin{aligned}
& {\left[\begin{array}{l}
x_{01} \\
x_{02} \\
x_{03} \\
u_{0} \\
u_{1}
\end{array}\right]=\left[\begin{array}{ccccc}
1 & 0 & 0 & 0 & 0 \\
0 & 0 & 1 & 1 & 0 \\
0 & 1 & 0 & 0 & 0 \\
1.12 & 1.8 & 1 & 0 & 0 \\
0 & 1.8 & 2.12 & 1 & 1
\end{array}\right]^{-1}\left[\begin{array}{c}
y_{01} \\
y_{02} \\
y_{12}-y_{02} \\
y_{21} \\
y_{22}
\end{array}\right]} \\
& =\left[\begin{array}{ccccc}
1 & 0 & 0 & 0 & 0 \\
0 & 0 & 1 & 0 & 0 \\
-1.12 & 0 & -1.8 & 1 & 0 \\
1.12 & 1 & 1.8 & -1 & 0 \\
1.254 & -1 & 0.216 & -1.12 & 1
\end{array}\right]\left[\begin{array}{c}
y_{01} \\
y_{02} \\
y_{12}-y_{02} \\
y_{21} \\
y_{22}
\end{array}\right] \\
& =\left[\begin{array}{c}
y_{01}-y_{02} \\
1.12 y_{01}+1.8 y_{12}-0.8 y_{02}-y_{21}
\end{array}\right]
\end{aligned}
$$

The solution (30) is unique since the matrix (25) has full column rank.

Case 2. $D \neq 0$.

Note that if $D \neq 0$ and $n>2$ then does not exist a nonsingular matrix $\bar{P} \in \mathfrak{R}^{n p \times n p}$ such that

$$
\bar{H}=\bar{P} \hat{H}
$$

where

$$
\hat{H}=\left[\begin{array}{cccccc}
C & D & 0 & \ldots & 0 & 0 \\
C A_{\alpha} & C B & D & \ldots & 0 & 0 \\
C A_{\alpha}^{2} & C A_{\alpha} B & C B & \ldots & 0 & 0 \\
\vdots & \vdots & \vdots & \ldots & \vdots & \vdots \\
C A_{\alpha}^{n-1} & C A_{\alpha}^{n-2} B & C A_{\alpha}^{n-3} B & \ldots & C B & D
\end{array}\right]
$$

and the matrix $\bar{H}$ is defined by (10b). For example

$$
\left[\begin{array}{cccc}
C & D & 0 & 0 \\
C \Phi_{1} & C B & D & 0 \\
C \Phi_{2} & C \Phi_{1} B & C B & D
\end{array}\right] \neq\left[\begin{array}{ccc}
I_{p} & 0 & 0 \\
0 & I_{p} & 0 \\
c_{1} I_{p} & 0 & I_{p}
\end{array}\right]\left[\begin{array}{cccc}
C & D & 0 & 0 \\
C A_{\alpha} & C B & D & 0 \\
C A_{\alpha}^{2} & C A_{\alpha} B & C B & D
\end{array}\right]
$$


Theorem 7 Let $D \neq 0$ and $m+1 \geqslant p$. Then the equation (10a) has the solution $x_{0}, u_{0}, u_{1}, \ldots, u_{n-1}$ for any given output sequence $y_{0}, y_{1}, \ldots, y_{n-1}$ if and only if

$$
\operatorname{rank} \bar{H}=p n .
$$

Moreover, the equation has the unique solution

$$
\bar{z}=\bar{H}^{-1} y
$$

if $m+1=p$ and it has many solutions if $m+1>p$.

Proof is similar to the proof of Theorem 4.

Theorem 8 Let $D \neq 0$ and $p>m+1$. Then the equation (10a) has a solution $x_{0}, u_{0}, u_{1}, \ldots, u_{n-1}$ for any given output sequence $y_{0}, y_{1}, \ldots, y_{n-1}$ if and only if the condition

$$
\operatorname{rank}\left[\begin{array}{ll}
\bar{H} & y
\end{array}\right]=\operatorname{rank} \bar{H}
$$

is met. Moreover, the equation has the unique solution if

$$
\operatorname{rank} \bar{H}=(m+1) n .
$$

Proof is similar to the proof of Theorem 5.

Theorem 9 The equation (10a) has unique solution only if the fractional system (1) is observable.

Proof The equation (10a) has the unique solution only if the matrix $\bar{H}$ has full column rank and this implies the full rank of the matrix $\bar{H}$.

Example 3 Consider the fractional system (1) for $\alpha=0.6$ with the matrices $A, B, C$ given by (24) and $D=\left[\begin{array}{l}0 \\ 1\end{array}\right]$. In this case the matrix

$$
\bar{H}=\left[\begin{array}{cccc}
C & D & 0 & 0 \\
C \Phi_{1} & C B & D & 0 \\
C \Phi_{2} & C \Phi_{1} B & C B & D
\end{array}\right]=\left[\begin{array}{cccccc}
1 & 0 & 0 & 0 & 0 & 0 \\
0 & 0 & 1 & 1 & 0 & 0 \\
1 & 1 & 0 & 0 & 0 & 0 \\
0 & 1 & 1 & 1 & 1 & 0 \\
1.12 & 1.8 & 1 & 0 & 0 & 0 \\
0 & 1.8 & 2.12 & 1 & 1 & 1
\end{array}\right]
$$


is nonsingular. Using (10a)) and (38) we obtain

$$
\begin{aligned}
& {\left[\begin{array}{l}
x_{01} \\
x_{02} \\
x_{03} \\
u_{0} \\
u_{1} \\
u_{2}
\end{array}\right]=\left[\begin{array}{cccccc}
1 & 0 & 0 & 0 & 0 & 0 \\
0 & 0 & 1 & 1 & 0 & 0 \\
1 & 1 & 0 & 0 & 0 & 0 \\
0 & 1 & 1 & 1 & 1 & 0 \\
1.12 & 1.8 & 1 & 0 & 0 & 0 \\
0 & 1.8 & 2.12 & 1 & 1 & 1
\end{array}\right]^{-1}\left[\begin{array}{l}
y_{01} \\
y_{02} \\
y_{11} \\
y_{12} \\
y_{21} \\
y_{22}
\end{array}\right]} \\
& =\left[\begin{array}{cccccc}
1 & 0 & 0 & 0 & 0 & 0 \\
-1 & 0 & 1 & 0 & 0 & 0 \\
0.68 & 0 & -1.8 & 0 & 1 & 0 \\
-0.68 & 1 & 1.8 & 0 & -1 & 0 \\
1 & -1 & -1 & 1 & 0 & 0 \\
0.038 & 0 & 1.216 & -1 & -1.12 & 1
\end{array}\right]\left[\begin{array}{l}
y_{01} \\
y_{02} \\
y_{11} \\
y_{12} \\
y_{21} \\
y_{22}
\end{array}\right] \\
& =\left[\begin{array}{c}
y_{01} \\
y_{11}-y_{01} \\
0.68 y_{01}-1.8 y_{11}+y_{21} \\
y_{02}+1.8 y_{11}-0.68 y_{01}-y_{21} \\
y_{01}-y_{02}-y_{11}+y_{12} \\
0.038 y_{01}+1.216 y_{11}-y_{12}-1.12 y_{21}+y_{22}
\end{array}\right] .
\end{aligned}
$$

\section{Positive systems}

Case 1. $D=0$.

From Definition 1 and Theorem 1 it follows that for positive fractional systems

$$
H \in \mathfrak{R}_{+}^{p n \times((m+1) n-m)}, \bar{H} \in \mathfrak{R}_{+}^{p n \times(n+1) m}, y \in \mathfrak{R}_{+}^{p n}, z \in \mathfrak{R}_{+}^{(m+1) n-m}, \bar{z} \in \mathfrak{R}_{+}^{(m+1) n}
$$

Definition 3 [3, 8] A square matrix A (a vector) is called the monomial matrix (vector) if its every row and its every column contains only one positive entry (one positive component) and the remaining entries (components) are zero.

Lemma 1 The inverse matrix $A^{-1}$ of a matrix $A \in \mathfrak{R}_{+}^{n \times n}$ is the positive matrix $A^{-1} \in \mathfrak{R}_{+}^{n \times n}$ if and only if $A$ is monomial matrix. 
Theorem 10 Let $D=0$ and $(m+1) n-m \geqslant n p$. Then the equation (9a) has a solution $x_{0} \in \mathfrak{R}_{+}^{n}, u_{i} \in \mathfrak{R}_{+}^{m}, i=0,1, \ldots, n-2$ for any given output sequence $y_{i} \in \mathfrak{R}_{+}^{p}$, $i=0,1, \ldots, n-1$ if and only if the following conditions are satisfied:

1. the condition (12) is met,

2. the matrix $H$ contains np linearly independent monomial columns.

Moreover, the equation has the unique solution

$$
z=H_{m}^{-1} y
$$

if the matrix $H$ contains only one monomial matrix $H_{m}$ and many solutions if it contains more than one such monomial matrices.

Proof The equation (9a) has a solution for any given sequence $y_{i} \in \mathfrak{R}_{+}^{p}, i=0,1, \ldots, n-1$ if and only if the condition (12) is met. By Lemma 1 the solution is nonnegative $x_{0} \in \Re_{+}^{n}$, $u_{i} \in \mathfrak{R}_{+}^{m}, i=0,1, \ldots, n-2$ for the output sequence if the matrix $H$ contains at least one monomial matrix $H_{m} \in \mathfrak{R}_{+}^{p n \times p n}$. The solution (41) is unique if the matrix $H$ contains only one monomial matrix and many solutions if it contains more than one such monomial matrices.

Theorem 11 Let $D=0$ and $n p>(m+1) n-m$. Then the equation (9a) has a solution $x_{0} \in \mathfrak{R}_{+}^{n}, u_{i} \in \mathfrak{R}_{+}^{m}, i=0,1, \ldots, n-2$ for any given output sequence $y_{i} \in \mathfrak{R}_{+}^{p}$, $i=0,1, \ldots, n-1$ if and only if the following conditions are satisfied:

1. the condition (17) is met,

2. the matrix $H^{\prime} \in \mathfrak{R}_{+}^{p n \times((m+1) n-m)}$ contains $(m+1) n-m$ linearly independent monomial rows.

Moreover, the equation has the unique solution

$$
z=\widetilde{H}_{m}^{-1} y
$$

where $\widetilde{H}_{m}$ is the monomial matrix consisting of linearly independent monomial rows

Proof For $n p>(m+1) n-m$ the equation (9a) has a solution if and only if the condition (17) is met. By Lemma 1 the solution is nonnegative $x_{0} \in \mathfrak{R}_{+}^{n}, u_{i} \in \mathfrak{R}_{+}^{m}, i=0,1, \ldots, n-2$ for a nonnegative sequence $y_{i} \in \mathfrak{R}_{+}^{p}, i=0,1, \ldots, n-1$ if and only if the matrix $H^{\prime}$ contains monomial matrix $\widetilde{H}_{m} \in \mathfrak{R}_{+}^{((m+1) n-m) \times((m+1) n-m)}$. The solution (42) is unique since the matrix $H^{\prime}$ contains only one monomial matrix $\widetilde{H}_{m}$.

Example 4 Consider the fractional positive system (1) for $\alpha=0.5$ and with the matrices

$$
A=\left[\begin{array}{ll}
0 & 0 \\
a & 1
\end{array}\right], B=\left[\begin{array}{l}
0 \\
1
\end{array}\right], C=\left[\begin{array}{ll}
0 & 1
\end{array}\right], D=[0]
$$


Find the values of the coefficient $a$ of $A$ for which it is possible to compute the initial condition $x_{0} \in \mathfrak{R}_{+}^{2}$ and the input $u_{0} \geqslant 0$ for a given output sequence $y_{i}>0, i=0,1$. In this case

$$
A_{\alpha}=A+I_{n} \alpha=\left[\begin{array}{cc}
0.5 & 0 \\
a & 1.5
\end{array}\right] \in \mathfrak{R}_{+}^{2 \times 2} \text { for } a \geqslant 0
$$

and the matrix

$$
H=\left[\begin{array}{cc}
C & 0 \\
C A & C B
\end{array}\right]=\left[\begin{array}{ccc}
0 & 1 & 0 \\
\alpha & 1 & 1
\end{array}\right] \in \mathfrak{R}_{+}^{2 \times 3}
$$

has only one linearly independent monomial column for all values of the coefficient $a$. The conditions of Theorem 10 are not satisfied and it is not possible to compute $x_{0} \in \mathfrak{R}_{+}^{2}$ and $u_{0} \geqslant 0$ for the given $y_{i} \geqslant 0, i=0,1$ for any values of the coefficient $a$. Note that the pair $\left(A_{\alpha}, C\right)$ is not observable for any values of $a$.

Case 1. $D \neq 0$.

Theorem 12 Let $D \neq 0$ and $m+1 \geqslant p$. Then the equation (10a) has a nonnegative solution $x_{0} \in \mathfrak{R}_{+}^{n}, u_{i} \in \mathfrak{R}_{+}^{m}, i=0,1, \ldots, n-1$ for any given output sequence $y_{i} \in \mathfrak{R}_{+}^{p}$, $i=0,1, \ldots, n-1$ if and only if the following conditions are satisfied:

1. the condition (34) is met,

2. the matrix $\bar{H} \in \mathfrak{R}_{+}^{p n \times((m+1) n-m)}$ contains $(m+1) n-m$ linearly independent monomial rows.

Moreover, the equation has the unique solution

$$
\bar{z}=\bar{H}_{m}^{-1} y
$$

if the matrix $\bar{H}$ contains only one monomial matrix $\bar{H}_{m}$ and it has many solutions if it contains more than one such monomial matrices.

Proof is similar to the proof of Theorem 10.

Theorem 13 Let $D \neq 0$ and $p>m+1$. Then the equation (10a) has a nonnegative solution $x_{0} \in \mathfrak{R}_{+}^{n}, u_{i} \in \mathfrak{R}_{+}^{m}, i=0,1, \ldots, n-1$ for any given output sequence $y_{i} \in \mathfrak{R}_{+}^{p}$, $i=0,1, \ldots, n-1$ if and only if the following conditions are satisfied:

1. the condition (17) is met,

2. the matrix $\bar{H} \in \mathfrak{R}_{+}^{p n \times(m+1) n}$ contains $(m+1) n$ linearly independent monomial rows. 
Proof is similar to the proof of Theorem 11.

Remark 1 The equation (10a) has the unique nonnegative solution only if the positive pair $\left(A_{\alpha}, C\right)$ is observable.

Example 5 Consider the positive fractional system (1) with $\alpha=0.5$ and the matrices

$$
A=\left[\begin{array}{ll}
0 & 0 \\
a & 1
\end{array}\right], B=\left[\begin{array}{l}
0 \\
1
\end{array}\right], C=\left[\begin{array}{ll}
1 & 0
\end{array}\right], D=[1]
$$

Find the values of the coefficient $a$ of $A$ for which it is possible to compute the initial condition $x_{0} \in \Re_{+}^{2}$ and the input $u_{0} \geqslant 0, i=0,1$ for a given output sequence $y_{i} \geqslant 0$, $i=0,1$. The matrix $A_{\alpha}$ is given by (44) and the matrix

$$
\bar{H}=\left[\begin{array}{ccc}
C & D & 0 \\
C A_{\alpha} & C B & D
\end{array}\right]=\left[\begin{array}{cccc}
1 & 0 & 1 & 0 \\
0.5 & 0 & 0 & 1
\end{array}\right]
$$

has two linearly independent monomial columns. The equation

$$
\left[\begin{array}{cccc}
1 & 0 & 1 & 0 \\
0.5 & 0 & 0 & 1
\end{array}\right]\left[\begin{array}{c}
x_{10} \\
x_{20} \\
u_{0} \\
u_{1}
\end{array}\right]=\left[\begin{array}{l}
y_{0} \\
y_{1}
\end{array}\right]
$$

has the solution $u_{0}=y_{0}, u_{1}=y_{1}$ for $x_{10}=0$ and arbitrary $x_{20}$ and any value of the coefficient $a \geqslant 0$.

\section{Concluding remarks}

The problem of computation of initial conditions and inputs for given outputs of the fractional standard and positive discrete-time linear systems has been formulated and solved. Two cases $D=0$ and $D \neq 0$ have been considered for standard and positive systems. Necessary and sufficient conditions have been established for existence of solution to the problem. It has been shown that there exist the unique solutions to the problem only if the pair $\left(A_{\alpha}, C\right)$ of the system is observable. The computation of initial conditions and inputs for given outputs can be considered as a generalized observability problem of fractional standard and positive linear systems. The considerations have been illustrated by numerical examples.

The considerations can be extended to the fractional standard and positive 2D linear systems. An extension of these considerations for continuous-time linear systems is an open problem. 


\section{References}

[1] P.J. Antsaklis and A.N. Michel: Linear Systems. Birkhauser, Boston 2006.

[2] L. FARINA and S. RinAlDi: Positive Linear Systems; Theory and Applications. J. Wiley, New York, 2000.

[3] T. KaczoreK: Positive 1D and 2D systems. Springer Verlag, London 2001.

[4] T. KaczoreK: Decomposition of the pairs $(\mathrm{A}, \mathrm{B})$ and $(\mathrm{A}, \mathrm{C})$ of the positive discrete-time linear systems. Archives of Control Sciences, 20(3), (2010), 253-273.

[5] T. KACZOREK: Vectors and Matrices in Automation and Electrotechnics. WNT, Warszawa, 1998, (in Polish).

[6] T. KaCzoreK: Linear Control Systems. Vol. 1, J. Wiley, New York, 1993.

[7] T. KaCzOREK: Computation of initial conditions and inputs for given output of standard and positive discrete-time linear systems. Computational Problems of Electrical Engineering, 2(1), (2012), (in press).

[8] T. KACZOREK: Selected Problems of Fractional System Theory. Spronger-Verlag, Berlin, 2011.

[9] T. KaILath: Linear Systems. Prentice-Hall, Englewood Cliffs, New York, 1980.

[10] R.E. Kalman: Mathematical Descriptions of Linear Systems. SIAM J. Control, 1 1963, 152-192.

[11] R.E. Kalman: On the General Theory of Control Systems. Proc. of the First Int. Congress on Automatic Control, Butterworth, London, 1960, 481-493.

[12] H.H. Rosenbrock: Comments on poles and zeros of linear multivariable systems: a survey of the algebraic geometric and complex variable theory. Int. J. Control, 26(1), 1977, 157-161.

[13] J. Klamka: Controllability of Dynamical Systems. Kluwer Academic Publisher, 1991.

[14] H.H. Rosenbrock: State-Space and Multivariable Theory. J. Wiley, New York, 1970.

[15] W.A. WolOvich: Linear Multivariable Systems. Springer-Verlag, New York, 1974. 